OPEN ACCESS

Edited by:

Hui Wu,

East China University of Science and Technology, China

Reviewed by:

Xinqing Zhao,

Shanghai Jiao Tong University, China

Estéfani García Ríos,

Consejo Superior de Investigaciones

Cientificas (CSIC), Spain

Soo Rin Kim,

Kyungpook National University,

South Korea

${ }^{*}$ Correspondence:

Yi Qin

qinyi@nwsuaf.edu.cn

Specialty section:

This article was submitted to Microbial Physiology and Metabolism,

a section of the journal

Frontiers in Microbiology

Received: 22 August 2020 Accepted: 23 November 2020 Published: 14 December 2020

Citation:

Du Q, Liu Y, Song Y and Qin Y (2020) Creation of a

Low-Alcohol-Production Yeast by

a Mutated SPT15 Transcription

Regulator Triggers Transcriptional and Metabolic Changes During Wine

Fermentation

Front. Microbiol. 11:597828. doi: 10.3389/fmicb.2020.597828

\section{Creation of a}

\section{Low-Alcohol-Production Yeast by a} Mutated SPT15 Transcription Regulator Triggers Transcriptional and Metabolic Changes During Wine Fermentation

\author{
Qing Du ${ }^{1,2,3}$, Yanlin Liu'1,2,3, Yuyang Song ${ }^{1,2,3}$ and Yi Qin 1,2,3* \\ ${ }^{1}$ College of Enology, Northwest A\&F University, Yangling, China, ${ }^{2}$ Shaanxi Engineering Research Center for Viti-Viniculture, \\ Yangling, China, ${ }^{3}$ National Forestry and Grassland Administration Engineering Research Center for Viti-Viniculture, Yangling, \\ China
}

There is significant interest in the wine industry to develop methods to reduce the ethanol content of wine. Here the global transcription machinery engineering (gTME) technology was used to engineer a yeast strain with decreased ethanol yield, based on the mutation of the SPT15 gene. We created a strain of Saccharomyces cerevisiae (YS59-409), which possessed ethanol yield reduced by $34.9 \%$; this was accompanied by the increase in $\mathrm{CO}_{2}$, biomass, and glycerol formation. Five mutation sites were identified in the mutated SPT15 gene of YS59-409. RNA-Seq and metabolome analysis of YS59-409 were conducted compared with control strain, suggesting that ribosome biogenesis, nucleotide metabolism, glycolysis flux, Crabtree effect, NAD ${ }^{+} / \mathrm{NADH}$ homeostasis and energy metabolism might be regulated by the mutagenesis of SPT15 gene. Furthermore, two genes related to energy metabolism, $R G / 1$ and $R G / 2$, were found to be associated with the weakened ethanol production capacity, although the precise mechanisms involved need to be further elucidated. This study highlighted the importance of applying gTME technology when attempting to reduce ethanol production by yeast, possibly reprogramming yeast's metabolism at the global level.

Keywords: low-alcohol, SPT15, RNA-seq, metabolome, wine, Saccharomyces cerevisiae

\section{INTRODUCTION}

Over recent decades, the alcohol concentration of wines produced by many warm regions around the world has increased by approximately $2 \%$ (v/v) (Goold et al., 2017). This is mainly due to the increasing preference of consumers for well-structured, full-bodied, and ripe-fruit wines and those wines are generally made from more mature grapes. Also, this has been exacerbated by global warming, which leads to higher sugar content in the grape varieties used to make wine 
(Varela et al., 2015; Rolle et al., 2017). Prompted by various reasons regarding wine quality, economic variables, and health concerns caused by high levels of alcohol, strategies aimed at reducing ethanol concentrations without impairing wine organoleptic quality have been performed in different ways (Varela et al., 2015; Dequin et al., 2017). Generally, microbiological strategies relating to the isolation and/or generation of the yeast strains used to make wine have proved to be the simplest and most economical methods, including Saccharomyces cerevisiae and non-conventional yeast species (Tilloy et al., 2015; Varela and Varela, 2018).

Engineering yeast strains with the capacity of redirecting carbon away from ethanol production to other endpoints is an effective approach and thus far, enhancing glycerol production has testified to be the most effective method (Otterstedt et al., 2004; Varela et al., 2012; Tan et al., 2016). Classical gene modification (GM) technologies have achieved increasing glycerol formation to reduce ethanol production by the manipulation of single or several genes. However, high concentrations of by-products, such as acetate, acetaldehyde, and acetoin, were generated in these previous experiments (Cambon et al., 2006; Varela et al., 2012). Since these by-products could have a significant negative effect on the flavor of wine, concerted efforts have been made to reduce the formation of these byproducts (Cambon et al., 2006; Ehsani et al., 2009). Additionally, a combination of adaptive evolution and breeding strategies is applied to develop a low-alcohol yeast for wine making with higher levels of glycerol production; this strain reduced ethanol production by $1.3 \%(\mathrm{v} / \mathrm{v})$ without the formation of undesirable by-products (Tilloy et al., 2014). Other researchers have focused on non-Saccharomyces strains in an attempt to reduce the ethanol content of wine; this is because such strains are known to exhibit different respiro-fermentative regulatory mechanisms when compared to S. cerevisiae (Quiros et al., 2014). Nevertheless, those non-Saccharomyces strains generally possess a weak capacity to complete wine fermentation on their own and must be accompanied by $S$. cerevisiae (Hranilovic et al., 2020). Consequently, there is significant interest in developing more effective strategies to balance low-ethanol wine production efficiency with good organoleptic qualities.

In this study, an alternative approach, global transcriptional machinery engineering (gTME), was used to develop strains of $S$. cerevisiae with reduced ethanol-production ability. The gTME technology was carried out by mutating the general transcription factor Spt15p, the TATA-binding protein (Alper et al., 2006); this protein plays a key role in the action of RNA polymerase and is one of the main DNA binding proteins that regulate promoter specificity in yeast (Eisenmann et al., 1989). The gTME technology is first used to improve the glucose/ethanol tolerance of $S$. cerevisiae, which shows the ability to re-program global gene transcription and change the complex phenotype of yeast strains (Alper et al., 2006; Alper and Stephanopoulos, 2007). Since then, several research studies have used the gTME approach to optimize the ethanol tolerance and ethanol production capacity of S. cerevisiae, and successfully demonstrated that gTME is advantageous when attempting to regulate the ethanol metabolism of yeast strains (Yang et al., 2011;
Seong et al., 2017). In the present study, we used gTME technology to weaken the capacity of yeast to produce ethanol and ultimately created a strain of S. cerevisiae (YS59-409) with a low yield of ethanol production. RNA-Seq and metabolomic analysis were also conducted in an attempt to understand the metabolic mechanisms underlying the modified phenotype of YS59-409. This study highlighted the critical role of the SPT15 regulator in reducing ethanol production in yeast and provided comprehensive insights to understand the molecular mechanisms of a new low-ethanol yeast.

\section{MATERIALS AND METHODS}

\section{Plasmids, Strains, SPT15 Mutant Library Construction and Culture Conditions}

We used S. cerevisiae YS59 (MAT $\alpha$; ura3-52, leu2-3, and his 5519) (Liu et al., 2007) as the host strain and then amplify the open reading frame of SPT15 gene from genomic DNA of YS59. The SPT15 gene was inserted into the restriction sites between $B a m \mathrm{HI}$ and EcoRI using the pY16 vector, which was flanked with TEF1 promoter and CYC1 terminator (pY16-SPT15); the plasmid has a URA3 selective marker, an ampicillin resistant marker and a $C E N / A R S$ element (low copy).

The yeast mutant library was created by random mutagenesis (error-prone PCR) of the SPT15 gene. Firstly, the SPT15 mutant library was generated using the Diversify ${ }^{\mathrm{TM}}$ PCR Random Mutagenesis kit (Clontech) with pY16-SPT15 as template. Plasmids obtained were transformed into Escherichia coli JM109 to produce a primary library for SPT15 mutants. From the sequencing of 20 randomly selected colonies, mutations were found at 2-10 sites without preference. Then library plasmids were transformed into S. cerevisiae YS59 and incubated at $25^{\circ} \mathrm{C}$ on solid SD to generate a yeast library for SPT15 mutant.

The plasmid was transformed into yeast cells by the lithium acetate method (Gietz and Woods, 2001). The strains and plasmids used in this procedure are summarized in Supplementary Table 1. S. cerevisiae strains were pre-cultured overnight in YPD medium (1\% yeast extract, $2 \%$ peptone, and $2 \%$ glucose) at $30^{\circ} \mathrm{C}$ for non-selective propagation. The selective culture of engineered strains was conducted in SD medium (0.67\% YNB, $0.077 \%$ Ura DO Supplement, and 2\% glucose) at $30^{\circ} \mathrm{C}$.

\section{Alcoholic Fermentation}

Alcoholic fermentation was carried out using an inoculum of $5 \times 10^{5}$ cells $/ \mathrm{mL}$ in a shaking incubator at $25^{\circ} \mathrm{C}$ at $150 \mathrm{rpm}$; all cultures were carried out in triplicate. The medium was similar to Triple $\mathrm{M}$ medium as reported previously (Spiropoulos et al., 2000), and consisted of $75 \mathrm{~g} / \mathrm{L}$ glucose, $75 \mathrm{~g} / \mathrm{L}$ fructose, $6 \mathrm{~g} / \mathrm{L}$ tartaric acid, $3 \mathrm{~g} / \mathrm{L}$ malic acid, $0.5 \mathrm{~g} / \mathrm{L}$ citric acid, $1.7 \mathrm{~g} / \mathrm{L}$ yeast nitrogen base without amino acids, $1 \mathrm{~g} / \mathrm{L}$ ammonium phosphate, $2 \mathrm{~g} / \mathrm{L}$ casamino acids, $0.8 \mathrm{~g} / \mathrm{L}$ L-arginine, $1 \mathrm{~g} / \mathrm{L} \mathrm{L}$ proline, $0.1 \mathrm{~g} / \mathrm{L}$ Tryptophan, and $4 \mathrm{~mL}$ ergo stock (composed of $250 \mathrm{~mL} / \mathrm{L}$ Tween $80,750 \mathrm{~mL} / \mathrm{L} 95 \%$ ethanol and $2.5 \mathrm{~g} / \mathrm{L}$ ergosterol), with $\mathrm{pH} 3.25$. 


\section{Site-Directed Mutagenesis of the SPT15 Gene and Fermentation Assays for Recombinant Strains}

Mutations in SPT15 in strain YS59-409 were identified by DNA sequencing. For site-directed mutagenesis, plasmid pY16-SPT15 (containing the non-mutated SPT15 gene) was used as a template with primers designed to the target nucleotide substitutions (Supplementary Table 2); these reactions were carried out with a Mut Express II Fast Mutagenesis Kit (Vazyme, China) in accordance with the manufacturer's directions. The reconstructed plasmids were then transformed into S. cerevisiae YS59 in order to obtain recombinant strains (Supplementary Table 1). The effects of mutation on the ethanol production capacity of strains were performed in Triple $\mathrm{M}$ media.

\section{RNA-Seq Analysis}

RNA-Seq analysis was used to investigate the transcriptional differences between the low-ethanol-production strain YS59409 and the control strain YS59-pY16. Three independent samples were collected from the mid-log phase of fermentation for RNA extraction. Total RNA was extracted using the procedures described previously (Li et al., 2019). Agarose gel (1\%) electrophoresis and a spectrophotometer (NanoDrop ND1000, United States) were then used to detect the purity and concentration of samples. Bioanalyzer (Agilent 2100, United States) was used to detect the RNA integrity numbers $(>8.0)$ of these samples to satisfy the particular requirements of RNA-seq. The cDNA library construction and RNA sequencing were carried out by Beijing Genomics Institute (Shenzhen, China) using standard protocols. The RNA-Seq data generated in this study were submitted to NCBI Sequence Read Archive (SRA) under the accession number PRJNA548495.

To compare the transcriptomes of the mutant and control yeast strains, we used Bowtie2 to map clean reads to the reference gene and then used HISAT to reference the genome of S. cerevisiae S288c. Gene expression levels were quantified using the FPKM method (Trapnell et al., 2012). Screening of differentially expressed genes (DEGs) between the two strains was conducted using the NOISeq method (Tarazona et al., 2011) based on a foldchange ( $\log _{2}$ Ratio) $\geq 1.5$ and a divergent probability $\geq 0.8$. Functional enrichment analysis of Gene Ontology (GO) and KEGG pathway enrichment analysis of DEGs were conducted by comparison with the entire genome background (Bonferroni-corrected $P$-value $<0.05$ ).

\section{Metabolomics Analysis}

The method used to prepare samples (six independent replicates) from the low-ethanol-production strain for metabolomic analysis was consistent with that used for RNA-seq analysis. Metabolites were extracted by vortex blending approximately $1 \mathrm{~g}$ of cells (fresh weight) in $1 \mathrm{~mL}$ of cold methyl alcohol for $30 \mathrm{~s}$. Cells were then lysed by ultrasonication for $15 \mathrm{~min}$ and centrifuged for $15 \mathrm{~min}$ at $12,000 \times g$ at $4^{\circ} \mathrm{C}$; an aliquot of $200 \mu \mathrm{L}$ of the supernatant was used for further analysis. Samples were analyzed by Shanghai Biocluster Biotech Co., Ltd. (China) using the Ultimate 3000 LC system coupled with an Orbitrap Elite mass spectrometer (Thermo, United States). The separation was performed on a Hypergod C18 column $(100 \mathrm{~mm} \times 4.6 \mathrm{~mm}$ $3 \mu \mathrm{m})$ at $40^{\circ} \mathrm{C}$. A flow rate of $0.3 \mathrm{~mL} / \mathrm{min}$, and an injection volume of $4 \mu \mathrm{L}$, were used in all analyses; this was followed by auto-sampling at $4^{\circ} \mathrm{C}$. Mass spectrometry detection used negative polarity with the following parameters: heater temperature, $300^{\circ} \mathrm{C}$; sheath gas flow rate, 45 arb; auxiliary gas flow rate, 15 arb; sweep gas flow rate, 1 arb; spray voltage, $3.2 \mathrm{kV}$; capillary temperature, $350^{\circ} \mathrm{C}$, and an S-Lens RF Level of $60 \%$.

With regards to multivariate statistical analysis, principal component analysis (PCA) and orthogonal partial least squares discriminant analysis (OPLS-DA) were performed using SIMCA$P$ version 13.0 software (Umetrics $A B$, Sweden) to separate the two groups of data. We then searched for differential metabolites using the variable importance in the projection (VIP) value of the OPLS-DA model (VIP > 1 ) in combination with the $p$-value of the $t$-test $(p<0.05)$. Qualitative metabolites were characterized by searching an online database ${ }^{1}$. Metabolic pathway analysis was carried out using MetaboAnalyst 3.02.

\section{Analytical Methods}

We measured the weight loss in $\mathrm{CO}_{2}$ from each sample by weighing the fermenters on a daily basis. Cell growth was recorded by a microplate reader at $\mathrm{OD}_{600} \mathrm{~nm}$ (BioTek, ELx800, United States). Ethanol yields were analyzed using an SBA-40C biological sensor analyzer (Biology Institute of Shandong Academy of Sciences, China). The content of reducing sugar in each sample was measured by the DNS method (Hu et al., 2008) with glucose as a standard using an ultra-violet spectrophotometer. Concentrations of glycerol and acetic acid were determined with an Enology Analyzer Y15 (BioSystems, Spain).

\section{Data Analysis}

Statistically significant differences between the wild-type (YS59pY16) and mutant yeast strains (YS59-409) were determined using the Student's $t$-test. The effect of site-directed mutagenesis and gene knockout on the fermentation characteristics of the two strains were further determined by one-way analysis of variance (ANOVA) and Duncan's test. The confidence level for both tests was 95\% and all analyses were carried out using SPSS version 19.0 software (SPSS Inc., United States).

\section{RESULTS AND DISCUSSION}

\section{Selection and the Characteristics of the Low-Ethanol-Yield Strain}

Previously, we produced a yeast mutant library ( $>1,000$ clones) that was based on S. cerevisiae YS59 and created by random mutagenesis (error-prone PCR) of the SPT15 gene with the pY16 plasmid backbone using gTME technology. Preliminary screening using SD media in 24-well plates was performed to identify the 20 mutants with the highest biomass; these were

\footnotetext{
${ }^{1}$ http://metlin.scripps.edu

${ }^{2}$ http://www.metaboanalyst.ca
} 
the strains that were supposed to have lower ethanol-production capacity due to competition for carbon sources between biomass and ethanol production (Supplementary Figure 1). To determine the ethanol production capacity of these 20 strains, we fermented each in 10-mL of Triple M media (approximately onefifth of the tube's total volume; this allowed the strains to grow fully) in the tubes. Finally, the strain with the lowest ethanol yield was identified (Supplementary Table 3). Compared with the control strain, the ethanol yield of the low-ethanol-production strain (YS59-409) was significantly reduced by 34.9\% (Table 1, $p<0.05)$. Interestingly, the strain featuring SPT15 gene mutation exhibited a greater $\mathrm{CO}_{2}$ weight loss than the control group (Figure 1). In the wine industry, $\mathrm{CO}_{2}$ weight loss is generally used as an indicator of the fermentation capacity of yeast. It is worth noting that the YS59-409 strain produced a larger amount of $\mathrm{CO}_{2}$ than the control but had a lower ethanol production. This suggests that other decarboxylation pathways might contribute to the $\mathrm{CO}_{2}$ loss in the mutant strain. In addition, the glycerol content of the YS59-409 strain was 43\% higher than the control strain, although there were no significant differences with regards to acetic acid production (Table 1). These findings were not consistent with the classical theory that higher glycerol synthesis is associated with increased acetic acid production (Nevoigt and Stahl, 1996). Commonly, the main by-product, glycerol, confers positive sensory effects, body, and sweetness, to wines when present in appropriate amounts and has been used as a target to redirect carbon sources away from ethanol production by many researchers in the wine industry (Tilloy et al., 2015; Goold et al., 2017). Our mutant strain (YS59-409) not only produced more glycerol than the control strain, but also did not accumulate overmuch acetic acid, an undesirable compound; these findings were also in agreement with those from previous studies (Ehsani et al., 2009; Tilloy et al., 2014). During wine fermentation, most of the sugars are used for the production of ethanol and $\mathrm{CO}_{2}$, with a small part for biomass and glycerol forming, including minute amounts of other byproducts. Therefore, we can speculate that strain YS59-409 uses more sugar for $\mathrm{CO}_{2}$, biomass, and glycerol synthesis, thus shunting the carbon source away from ethanol synthesis and resulting in low ethanol yield.

\section{Sequence Analysis of the SPT15 Gene in the YS59-409 Strain}

The SPT15 gene in YS59-409 was amplified from the pY16-409 plasmid and then was sequenced. There were 5 mutational sites in the structural domain of the mutated SPT15 gene as shown in Figure 2, in which methionine is substituted for isoleucine (Ile ${ }^{46} \mathrm{Met}$ ), and similarly, Asp ${ }^{56} \mathrm{Gly}$, Ser ${ }^{118} \mathrm{Pro}$, Tyr ${ }^{195} \mathrm{His}$, and Leu $^{205}$ Ser (I46M, D56G, S118P, Y195H, and L205S, respectively). Three point mutations were located in the highly conserved domain (amino acids 61-240) and two mutations were located in the non-conserved domain (amino acids 1-60). To explore which of these sites conferred the most desirable phenotype to the low-ethanol-yielding strain, we constructed five strains featuring single point mutations (Supplementary Table 1). However, none of these mutants led to a reduction in ethanol yield; instead, I46M, D56G, and L205S, led to enhanced ethanol yield compared with the control under fermentation conditions (Table 2). Of all these mutational sites, Y195H has been reported to work together with F177S and K218R to improve glucose/ethanol tolerance and the efficiency of glucose conversion to ethanol of yeast (Alper et al., 2006); and Leu-205 site has shown to be important for DNA binding specificity of Spt15p and mutating this site to other different amino acids can cause various degrees of changes in yeast growth (Arndt et al., 1992). Although our results showed that single mutation of Y195H or L205S did not produce the lowalcohol phenotype, we hypothesized that these two sites might play key roles in the mutant strain and that the desired phenotype might be obtained through double or multiple mutations based on previous reports (Arndt et al., 1992; Alper et al., 2006). In addition, the same mutation on SPT15 may lead to different phenotypes in strains with different genetic backgrounds. In a word, these results illustrate that the combined action of the five separate mutations, or at least in part, conferred the desired phenotype to the mutant strain.

\section{Transcriptional and Metabolic Analysis of the Mutated Strain}

As described in previous research, gTME can trigger overall disturbances at the transcriptional level and can be used to unravel complex phenotypes (Alper and Stephanopoulos, 2007). In order to investigate the mechanisms underlying the observed phenotype, we used RNA-Seq and metabolomic analysis to analyze the differences in transcription and metabolism between mutant strain (YS59-409) and the control (YS59-pY16). Under fermentation conditions, we observed significant changes in the transcription and metabolism of the mutant; these factors are likely to have made an important contribution to the target phenotype of the mutant. Subsequently, we would discuss the overall transcriptional and metabolic regulation and the specific pathways and function change associated with ethanol metabolism answering to the mutation of SPT15 gene.

\section{The Overall Analysis of Transcription and Metabolism in Strain YS59-409}

A total of 964 genes showed significantly different expression levels when compared between the mutant and control strains; 636 genes were up-regulated and 328 genes were down-regulated in the mutant YS59-409 strain (Supplementary Table 4). These differentially expressed genes (DEGs) demonstrated that the mutation of the SPT15 gene, a transcription factor, led to the global reprogramming of transcription at genes and may have contributed to the regulation of ethanol metabolism in this mutant strain. GO analysis and KEGG pathway enrichment analysis were used to further analyze the functional enrichment of DEGs. KEGG pathway enrichment analysis revealed that most of the DEGs participated in those key metabolic pathways, including translation, transcription, carbohydrate metabolism, nucleotide metabolism, and amino acid metabolism (Table 3).

There were 41 differential metabolites identified (16 upregulated and 25 downregulated), as shown in Supplementary Table 6. OPLS-DA and PCA analysis were conducted to identify differences in the metabolome that were caused by random mutagenesis of the SPT15 gene. We found there 
TABLE 1 | Fermentation characteristics of the mutant strain S. cerevisiae YS59-409.

\begin{tabular}{|c|c|c|c|c|c|c|c|}
\hline Strains & $O D_{600}$ & Residual sugar (g/L) & Ethanol (g/L) & Glycerol (g/L) & Acetate (g/L) & Ethanol yield ${ }^{a}$ (g/g Sugar) & $\begin{array}{c}\text { Change in ethanol } \\
\text { yield }\end{array}$ \\
\hline YS59-pY16 & $4.52 \pm 0.34$ & $1.13 \pm 0.35$ & $54.67 \pm 0.94$ & $2.13 \pm 0.08$ & $0.493 \pm 0.109$ & $0.367 \pm 0.007$ & 0 \\
\hline YS59-409 & $5.45 \pm 0.40^{\star}$ & $1.90 \pm 0.30$ & $35.33 \pm 3.09^{\star}$ & $3.05 \pm 0.07^{\star}$ & $0.487 \pm 0.066$ & $0.239 \pm 0.021^{\star}$ & $-34.9 \%$ \\
\hline
\end{tabular}

${ }^{a}$ Ethanol yield represents the ratio of ethanol production $(g)$ to the sugar consumption $(g)$.

*Student's t-test showed significant difference at the level of 0.05.

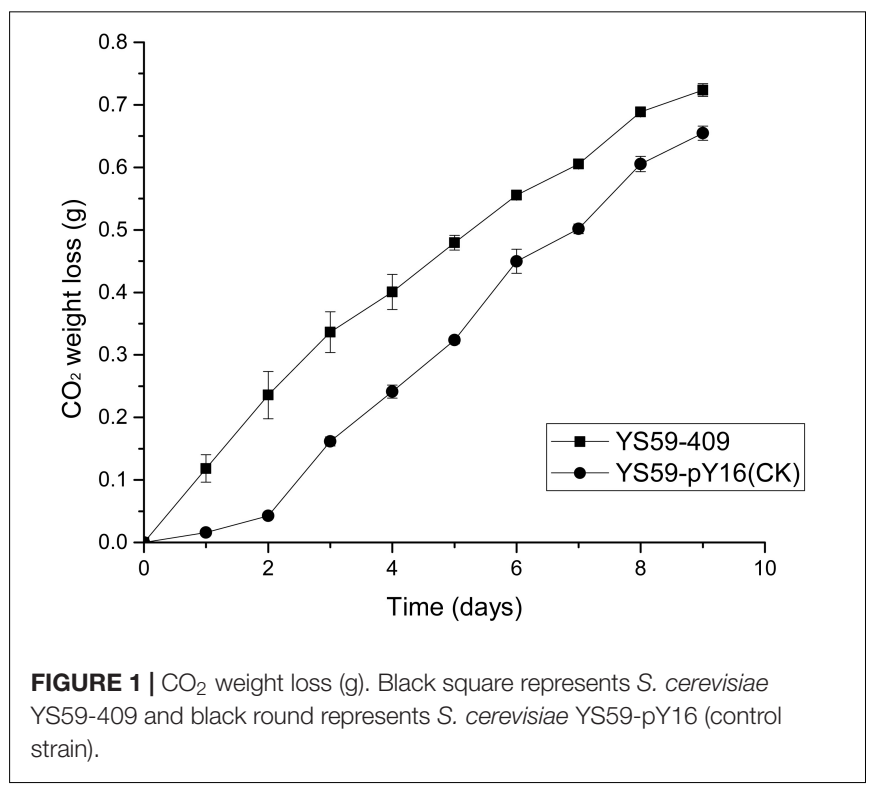

were distinct discrepancies between the mutant and control strains in terms of metabolic profile (Supplementary Figure 2). Indeed, we found that several metabolites related to amino acid metabolism (L-glutamine, L-glutamate, L-tryptophan, L-histidine, L-lysine, kynurenine, glutathione, succinic acid semialdehyde, and adenylosuccinate) (Supplementary Table 6) showed significant differences when compared between the two strains, corresponding to transcriptional alterations in amino acid metabolism.

\section{Transcription, Translation, and Nucleotide Metabolism, Were Activated in Strain YS59-409}

In total, 14 and 135 genes were functionally annotated into transcription and translation, respectively (Table 3 and Supplementary Tables 4, 5). Genes related to RNA polymerase were upregulated, thus indicating the transcriptional activation of the mutant strain. This could be explained by the upregulation of SPT15 in the mutant YS59-409 strain (Supplementary Table 4) for its regulatory effect on RNA polymerase and gene transcription levels (Eisenmann et al., 1989). In addition, $R P L$ and RPS genes encoding ribosome biogenesis proteins were significantly over-expressed in the low ethanol-production strain (Supplementary Tables 4, 5), which reflected the stimulation of relative translational activity at certain time points (Backhus et al., 2001). Coincidently, the upregulation of RNA polymerase metabolism and ribosome biogenesis were reported in a low-ethanol-production yeast by a previous study (Varela et al., 2018). Furthermore, most of the genes associated with nucleotide metabolism were expressed at high levels (Table 3 and Supplementary Tables 4, 5), of which purines and pyrimidines are the basic components (Ljungdahl and Daignan-Fornier, 2012), exhibiting the activation of purine and pyrimidine metabolism. Collectively, these results above indicate that transcription, translation, and nucleotide metabolism, were activated in the mutant YS59-409 strain. This probably demonstrates that the mutation of the SPT15 gene leads to the reprogramming of global gene expression and metabolism.

\section{The Fermentative Pathways Leading to Ethanol Production Was Reduced in Strain YS59-409}

Glycolytic flux relies on glucose uptake rate, which is regulated by a family of hexose transporters encoded by HXT genes. In the present study, we found that several hexose transporter genes were significantly downregulated in the YS59-409 strain (HXT2, HXT4, HXT5, HXT6, HXT7, HXT9, HXT11, and HXT13; Figure 3 and Supplementary Table 4); of these, HXT4, HXT6, and HXT7, are known to be vital for the uptake of glucose (Özcan and Johnston, 1999). Previous research has reported that a deficiency of hexose transporters in S. cerevisiae could reduce the levels of sugar uptake and thus regulate the glycolysis flux, particularly with regards to HXT7, which eventually resulted in a reduction in ethanol production (Otterstedt et al., 2004). Consequently, the significant downregulation of several HXT genes in YS59-409 is likely to result in a decrease of glycolysis flux. Besides, the expression of HXK1 was downregulated by 4.7-fold (Figure 3

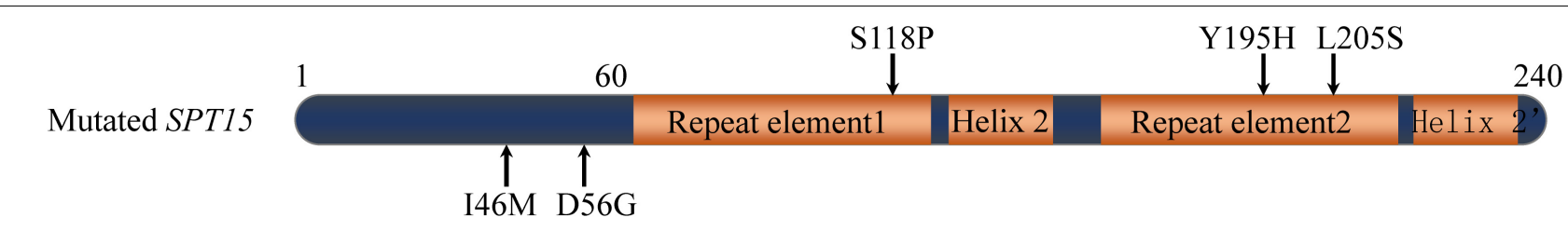

FIGURE 2 | Mutation sites in the SPT15 gene of mutant strain (arrows). The schematic of structural domain is referred to the previous study (Alper et al., 2006). 
TABLE 2 | Fermentation characteristics of point-mutated strains.

\begin{tabular}{|c|c|c|c|c|}
\hline Strains & Residual sugar (g/L) & Ethanol (g/L) & Ethanol yield (g/g sugar) & Changes in ethanol yield \\
\hline $409-46$ & $0.56 \pm 0.91^{a}$ & $53.09 \pm 0.36^{b}$ & $0.348 \pm 0.005^{\mathrm{ac}}$ & $8.10 \%$ \\
\hline $409-56$ & $1.10 \pm 1.67^{a}$ & $52.23 \pm 1.09^{b}$ & $0.357 \pm 0.018^{a}$ & $11.00 \%$ \\
\hline $409-118$ & $1.04 \pm 0.01^{a}$ & $51.42 \pm 1.76^{a b}$ & $0.325 \pm 0.005^{\mathrm{b}}$ & $1.10 \%$ \\
\hline 409-195 & $1.03 \pm 0.02^{a}$ & $50.19 \pm 0.49^{a b}$ & $0.332 \pm 0.003^{b c}$ & $3.10 \%$ \\
\hline 409-205 & $1.03 \pm 0.02^{a}$ & $53.34 \pm 3.19^{b}$ & $0.355 \pm 0.013^{a}$ & $10.40 \%$ \\
\hline YS59-pY16 & $1.02 \pm 0.02^{a}$ & $48.43 \pm 0.24^{a}$ & $0.322 \pm 0.003^{b}$ & 0 \\
\hline
\end{tabular}

${ }^{a, b, c}$ Different letters indicate significant differences according to the Duncan test $(p<0.05)$. Data are mean $\pm S D$ of independent triplicate.

and Supplementary Table 4); this gene encodes hexokinase 1 , which is responsible for catalyzing the phosphorylation of glucose in the first irreversible step of glycolysis (Rodríguez et al., 2001). The downregulation of $H X K 1$ also might contribute to the reduced glycolysis metabolism in the mutant; this observation is supported by a previous study that the repression of hexokinase activity resulted in reduced glycolysis flux (Tan et al., 2016). So, the downregulation of HXTs and HXK1 in strain YS59409 probably lead to a reduction in glycolysis flux compared to the control strain, thus well explaining the reduced yield of ethanol. Varela et al. (2018) have reported that the low ethanol strain produced in their study exhibited reduced glycolysis activity, which agrees well with the present study. What's more, a reduction in pyruvate content (Figure 3 and Supplementary Table 6) provides further evidence of the weaker glycolysis flux (Otterstedt et al., 2004). We hypothesize that the glycolysis flux of the YS59-409 strain is reduced by mutation of the SPT15 gene, thus resulting in a weakened ethanol fermentation pathway of the YS59-409 strain.

\section{The Low-Ethanol-Producing Strain YS59-409 Exhibited a Disturbance in the Crabtree Effect}

Saccharomyces cerevisiae is a Crabtree-positive yeast, which generally utilizes the ethanol fermentation pathway for glucose

TABLE 3 | KEGG pathway enrichment analysis ( $p$-value $<0.05)$.

\begin{tabular}{lccc}
\hline Pathway & P-value & $\begin{array}{c}\text { Number of } \\
\text { genes }\end{array}$ & $\begin{array}{c}\text { Gene match } \\
\text { (genome match) }\end{array}$ \\
\hline $\begin{array}{l}\text { Translation } \\
\text { Ribosome }\end{array}$ & $1.00 \mathrm{E}-71$ & 135 & $19.77(4.33)$ \\
$\begin{array}{l}\text { Transcription } \\
\text { RNA polymerase }\end{array}$ & $5.87 \mathrm{E}-05$ & 14 & $2.05(0.69)$ \\
$\begin{array}{l}\text { Carbohydrate metabolism } \\
\text { Starch and sucrose metabolism }\end{array}$ & $6.27 \mathrm{E}-10$ & 22 & $3.22(0.84)$ \\
$\begin{array}{l}\text { Galactose metabolism } \\
\text { Nucleotide metabolism }\end{array}$ & $6.11 \mathrm{E}-04$ & 13 & $1.90(0.74)$ \\
$\begin{array}{l}\text { Pyrimidine metabolism } \\
\text { Purine metabolism }\end{array}$ & $1.28 \mathrm{E}-04$ & 24 & $3.51(1.65)$ \\
$\begin{array}{l}\text { Amino acid metabolism } \\
\text { Histidine metabolism }\end{array}$ & $3.23 \mathrm{E}-02$ & 24 & $3.51(2.41)$ \\
Arginine and proline metabolism & $4.58 \mathrm{E}-02$ & 6 & $0.88(0.26)$ \\
\hline
\end{tabular}

a The percentage of DEGs involved in individual pathway account for all DEGs with pathway annotation (683) and all genes with pathway annotation (4184). consumption under conditions of excess glucose, even in the presence of oxygen (also referred to as 'overflow metabolism') (de Deken, 1966). In the present study, we observed indications that the Crabtree effect of the YS59-409 strain might be disturbed. On the one hand, the attenuated glycolysis flux in the YS59-409 strain, in combination with the higher $\mathrm{CO}_{2}$ production and the lower ethanol formation rate, appeared to suggest that the TCA cycle was enhanced. This deduction could be supported by an earlier study showing that when glucose uptake rates were reduced, then the $\mathrm{CO}_{2}$ /ethanol ratio increased more than $50 \%$ and the net flux through the TCA cycle increased significantly (Heyland et al., 2009). That is to say, the overflow metabolism of the YS59-409 strain might shift toward respiratory metabolism. Our specific experimental conditions (the headspace of the 24-well plates and test tubes) could provide a micro-oxygen environment that can support the respiration of yeast to some extent, as also noted by a previous study (Heyland et al., 2009). Respiratory metabolism plays an important role in terms of producing energy in the form of ATP in aerobic growing cells. Intriguingly, we found that the energetic metabolism of YS59-409 may be enhanced compared to the control strain. As is well-known, the histidine and nucleotide biosynthetic pathways are connected (Ljungdahl and Daignan-Fornier, 2012). The upregulation of genes related to histidine metabolism (such as HIS1, HIS2, HIS5, and HIS7), accompanied by the increased histidine content in YS59-409 (Figure 3), provided strong evidence for an enhancement in the synthesis of histidine. And KEGG pathway enrichment analysis indicated that purine synthesis metabolism was reinforced in the mutant strain (Table 3 and Supplementary Tables 4, 5). So, with the viewpoint that the de novo purine pathway feeds into the histidine pathway and branches to allow ATP synthesis (Ljungdahl and Daignan-Fornier, 2012), we can speculate that ATP production in the YS59-409 strain is boosted. In addition, the upregulation of the $A D E 4$ gene in the first step of the ATP formation pathway (Ljungdahl and Daignan-Fornier, 2012) provided supplementary evidence to the enhanced ATP synthesis. What's more, high ATP yields may result in excess biomass formation at the expense of product yield (de Kok et al., 2012), which further supports the fact that YS59-409 showed higher biomasses and lower ethanol production. Therefore, we can infer that the characteristic of Crabtree effect of the mutant strain have been changed. On the other hand, some Crabtreenegative yeast, such as Hanseniaspora uvarum and Metschnikowia pulcherrima, possess different respiro-fermentative regulatory 


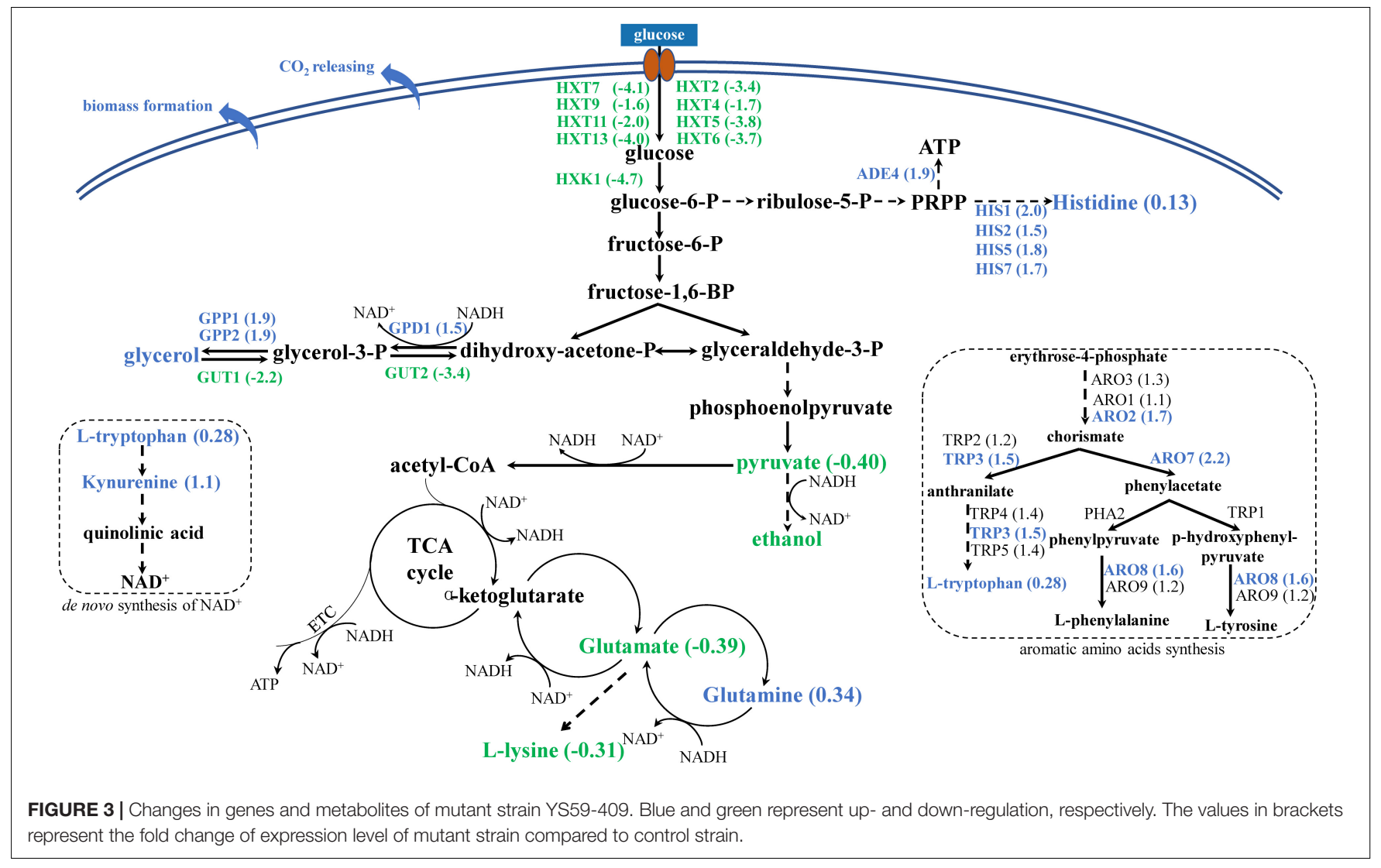

mechanisms than S. cerevisiae (Quiros et al., 2014). Researchers have used these non-conventional species to reduce ethanol production by partial controlling the aeration of grape juice (Quiros et al., 2014). However, non-conventional yeasts cannot generally complete alcoholic fermentation. We created a new mutant strain (YS59-409), which might not only have similar ethanol-producing properties as non-conventional yeasts, but also is capable of finishing fermentation alone. Thus, these results provide a new concept for the creation of new low-ethanolproduction strains.

\section{NAD + /NADH Homeostasis Was Disturbed in Strain YS59-409}

Sugar fermentation in $S$. cerevisiae is a redox neutral process that is influenced by $\mathrm{NAD}^{+} / \mathrm{NADH}$ balance, in which glycerol plays important roles (Goold et al., 2017). We found those key genes in the synthesis pathway of glycerol, GPD1, encoding glycerol-3-phosphate dehydrogenase, along with GPP1 and GPP2, encoding glycerol-3-phosphate phosphatase (Nevoigt and Stahl, 1997), were overexpressed in the low-ethanolproducing strain (Figure 3 and Supplementary Table 4). Simultaneously, GUT1 and GUT2, genes that encode for glycerol kinase for glycerol catabolism (Nevoigt and Stahl, 1997) were both downregulated (Figure 3 and Supplementary Table 4). Changes in the expression of those genes related to glycerol metabolism demonstrated the high production of glycerol; this was confirmed by the increased glycerol concentration in the

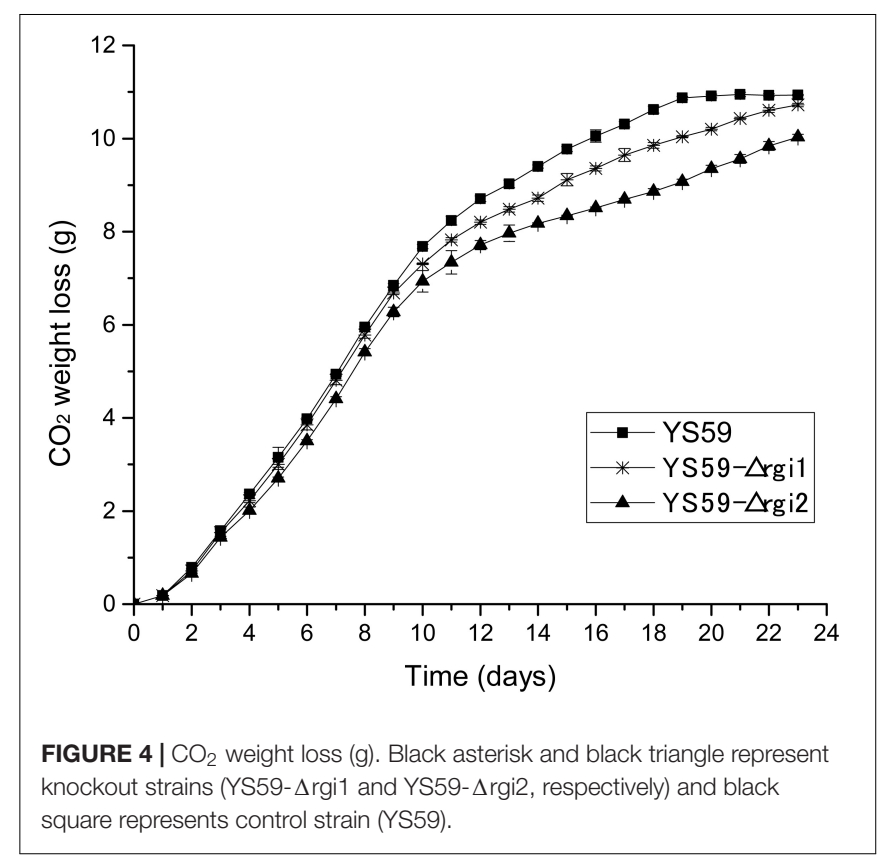

YS59-409 strain (Table 1). Higher production of glycerol is likely due to the need to balance cytosolic NADH produced and consumed. What is noticeable is that the de novo pathway of $\mathrm{NAD}^{+}$(namely, the kynurenine pathway) might 
TABLE 4 | Fermentation characteristics of knockout strains.

\begin{tabular}{|c|c|c|c|c|}
\hline Strains & Residual sugar (g/L) & Ethanol (g/L) & Ethanol yield (g/g Sugar) & Change in ethanol yield \\
\hline YS59 & $1.96 \pm 0.68^{a}$ & $52.04 \pm 1.43^{c}$ & $0.351 \pm 0.008^{c}$ & 0 \\
\hline YS59- $\Delta$ rgi1 & $0.99 \pm 0.19^{a}$ & $40.59 \pm 0.96^{a}$ & $0.272 \pm 0.006^{\mathrm{a}}$ & $-22.0 \%$ \\
\hline YS59- $\Delta$ rgi2 & $1.62 \pm 0.40^{\mathrm{a}}$ & $43.43 \pm 0.77^{b}$ & $0.292 \pm 0.004^{b}$ & $-16.5 \%$ \\
\hline
\end{tabular}

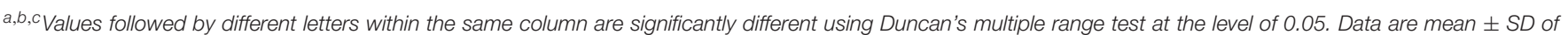
independent triplicate.

have been disturbed. In yeast, $\mathrm{NAD}^{+}$can be synthesized de novo from tryptophan (Panozzoa et al., 2002). We found that tryptophan content was increased in the mutant strain (Figure 3 and Supplementary Table 6); this was consistent with the fact that genes involved in the synthesis of tryptophan were also increased (Figure 3 and Supplementary Table 4). Moreover, as the key participator in the de novo synthesis of $\mathrm{NAD}^{+}$from L-tryptophan (Panozzoa et al., 2002), kynurenine was found to be the increased metabolite with the highest fold-change in this study (Figure 3 and Supplementary Table 6). Previous studies have shown that the de novo pathway plays only a minor role if a functional salvage pathway is present (Sporty et al., 2009); and one of the key requirements in the formation of kynurenine is that the kynurenine pathway needs oxygen (Panozzoa et al., 2002). Therefore, the increased production of kynurenine and Ltryptophan in the mutant strain probably shows the activation of the de novo pathway for the synthesis of $\mathrm{NAD}^{+}$under our experimental conditions. The strengthening trend of $\mathrm{NAD}^{+}$ level might further explain the enhanced synthesis of ATP synthesis for the reason that ATP synthesis and redox potential are directly proportional to the intracellular concentration of $\mathrm{NAD}^{+}$(Gonzalez Esquivel et al., 2017). Collectively, those data indicate that $\mathrm{NAD}^{+} / \mathrm{NADH}$ equilibrium might be disturbed in the mutant strain. Certainly, the $\mathrm{NAD}^{+} / \mathrm{NADH}$ balance of the YS59-409 strain depends on a range of factors, including biomass formation, respiration, ATP production, and the generation of some metabolites, such as ethanol, glycerol, and amino acids. Additionally, researchers have deployed methods to intentionally perturb the $\mathrm{NAD}^{+} / \mathrm{NADH}$ balance to reduce ethanol production (Goold et al., 2017), which also emphasizes the importance of $\mathrm{NAD}^{+} / \mathrm{NADH}$ balance for ethanol metabolism.

\section{The Effects of Deleting the RGI1/2 Gene on the Ethanol-Production Capacity of Yeast}

To further identify key genes in the regulatory network of our new mutant strain, we constructed a protein-protein interaction (PPI) network using the STRING database V1 $11^{3}$ and Cytoscape tools; the core gene module was then excavated using the MCODE plugin in Cytoscape. Nine genes were identified (Supplementary Table 7); of these, AQY3 (Yfl054c) and RGI2 (Yil057c) were shown to be associated with glycerol and energy metabolism, which was corresponded with the changes in

${ }^{3}$ https://string-db.org/ glycerol and energy metabolism observed in YS59-409. Then, we found that only RGI2 exerted influence over ethanol production. The RGI2 gene possesses very little data concerning protein function or biological processes involved for itself, which was found to be significantly down-regulated by 41.9-fold in this study (Supplementary Table 4). Noteworthily, the homologous gene of RGI2, RGI1 (Yer067w), was significantly down-regulated by 4.3 -fold (Supplementary Table 4), which shared 70\% identity with the sequence of RGI2 (Domitrovic et al., 2010). RGI1 gene is reported to be regulated transcriptionally by SPT15 under conditions involving ethanol stress (Yang et al., 2011). Previous studies show that Rgilp and Rgi2p proteins most likely belong to the same complex and/or operate in the same pathway, and that these proteins are involved in the control of energetic metabolism, particularly under respiratory growth conditions (Domitrovic et al., 2010). Therefore, the effects of those two genes on ethanol metabolism were conducted in the present study. We performed single gene knock-outs for RGI1 and RGI2 genes in strain YS59 using Cre/loxP-mediated technology in order to evaluate their effects on ethanol production in $S$. cerevisiae, generating two new strains: YS59- $\Delta$ rgi1 and YS59$\Delta$ rgi2 (Supplementary Table 1). As follow, we compared the performance of strain YS59- $\Delta$ rgil and strain YS59- $\Delta$ rgi2 in Triple M media (250-mL flasks containing $150-\mathrm{mL}$ of media). Compared with the control strain YS59, the weight loss of $\mathrm{CO}_{2}$ was both reduced in the two knockout strains (Figure 4), thus illustrating their reduced fermentation capacity; this was further supported by the fact that these strains showed a 22.0 and $16.5 \%$ reduction in ethanol productivities, respectively (Table 4). The results implied that perturbation of the RGI1 or RGI2 gene could elicit alterations in ethanol metabolism, which showed the positive effects of $R G I 1 / 2$ deletion with regards to weakening the ethanol-yielding ability of yeast. However, these two knockout strains may have a different low-yield ethanol mechanism than the YS59-409 strain, because they had different $\mathrm{CO}_{2}$ production models; this possibility requires further investigation.

\section{CONCLUSION}

In this study, we applied gTME technology to engineer a lowethanol-production strain of $S$. cerevisiae achieved by mutating the transcription factor SPT15 to change gene expression in a global level. We provide a novel insight into the use of gTME technology to modulate ethanol metabolism, which could not only facilitate the construction of a low-ethanol-production strain for the wine industry, but also, enhance our understanding 
of the mechanisms underlying reduced ethanol production by yeast. We created a new strain of S. cerevisiae, YS59-409, with weakened ethanol production capacity. The ethanol-production capacity of this strain was reduced by $34.9 \%$ compared to the control strain, which was caused by comprehensive changes associated with the regulation of transcription and metabolism. Sequence analysis was performed on the mutated SPT15 gene, demonstrating that the five mutation sites may work collectively, or at least partly, to create the specific characteristics of YS59-409, including a higher $\mathrm{CO}_{2}$ release, biomass, and glycerol formation. The integration of RNA-Seq and metabolomics analysis showed that the specific phenotype of the new mutant strain featured changes in ribosome biogenesis, nucleotide metabolism, glycolysis flux, the Crabtree effect, $\mathrm{NAD}^{+} / \mathrm{NADH}$ homeostasis, and energy metabolism. Remarkably, we also found that RGI1 and RGI2 genes, which play key roles in energy metabolism, were significantly down-regulated; it is possible that this was linked with low ethanol metabolism, although this needs to be investigated further in future research. Although public attitudes toward the use of GMOs (Genetically Modified Organisms) in wines are often less than positive, this study demonstrated that it is possible to reduce ethanol yields in yeast using gTME technology, while also reprogramming the metabolism of this new mutant strain. Currently, we can get some knowledge from this study, which could be used to direct strategies for generating wine yeast with weakened ethanol production capacity using other approaches, such as adaptive evolution. In summary, this study highlights the potential to use gTME technology to reduce the ethanol content of yeast for the winemaking industry.

\section{REFERENCES}

Alper, H., Moxley, J., Nevoigt, E., Fink, G. R., and Stephanopoulos, G. (2006). Engineering yeast transcription machinery for improved ethanol tolerance and production. Science 314, 1565-1568. doi: 10.1126/science.1131969

Alper, H., and Stephanopoulos, G. (2007). Global transcription machinery engineering: A new approach for improving cellular phenotype. Metab. Eng. 9, 258-267. doi: 10.1016/j.ymben.2006.12.002

Arndt, K. M., Ricupero, S. L., Eisenmann, D. M., and Winston, F. (1992). Biochemical and genetic characterization of a yeast TFIID mutant that alters transcription in vivo and DNA binding in vitro. Molecular and Cellular Biology 12, 2372-2382. doi: $10.1128 / \mathrm{mcb} .12 .5 .2372$

Backhus, L. E., Derisi, J., Brown, P. O., and Bisson, L. F. (2001). Functional genomic analysis of a commercial wine strain of Saccharomyces cerevisiae under differing nitrogen conditions. FEMS Yeast Res. 1, 111-125. doi: 10.1111/j.1567-1364. 2001.tb00022.x

Cambon, B., Monteil, V., Remize, F., Camarasa, C., and Dequin, S. (2006). Effects of GPD1 overexpression in Saccharomyces cerevisiae commercial wine yeast strains lacking ALD6 genes. Appl. Environ. Microbiol. 72, 4688-4694. doi: 10. 1128/AEM.02975-05

de Deken, R. H. (1966). The crabtree effect: A regulatory system in yeast. J. Gen. Microbiol. 44, 149-156. doi: 10.1099/00221287-44-2-149

de Kok, S., Kozak, B. U., Pronk, J. T., and van Maris, A. J. (2012). Energy coupling in Saccharomyces cerevisiae: Selected opportunities for metabolic engineering. FEMS Yeast Res. 12, 387-397. doi: 10.1111/j.1567-1364.2012.00799.x

Dequin, S., Escudier, J. L., Bely, M., Noble, J., Albertin, W., Masneuf-Pomarède, I., et al. (2017). How to adapt winemaking practices to modified grape

\section{DATA AVAILABILITY STATEMENT}

The datasets generated for this study can be found in online repositories. The names of the repository/repositories and accession number(s) can be found in the article/ Supplementary Material.

\section{AUTHOR CONTRIBUTIONS}

QD: experimental operation and writing - original draft. YL: supervision. YS: data analysis. YQ: experimental design and supervision. All authors edited and approved the final version of the manuscript.

\section{FUNDING}

This project was supported by National Key Research and Development Project (2019YFD1002500), National Natural Science Foundation of China (31301541 and 31960470), the Fundamental Research Funds for the Central Universities (2452020177), and China Agriculture Research System (grant no. CARS-29-jg-3).

\section{SUPPLEMENTARY MATERIAL}

The Supplementary Material for this article can be found online at: https://www.frontiersin.org/articles/10.3389/fmicb. 2020.597828/full\#supplementary-material

composition under climate change conditions. Oeno One 51, 205. doi: 10.20870/ oeno-one.2016.0.0.1584

Domitrovic, T., Kozlov, G., Freire, J. C., Masuda, C. A., Almeida, M., MonteroLomeli, M., et al. (2010). Structural and functional study of Yer067w, a new protein involved in yeast metabolism control and drug resistance. PLoS One 5:e11163. doi: 10.1371/journal.pone.0011163

Ehsani, M., Fernandez, M. R., Biosca, J. A., Julien, A., and Dequin, S. (2009). Engineering of 2,3-butanediol dehydrogenase to reduce acetoin formation by glycerol-overproducing, low-alcohol Saccharomyces cerevisiae. Appl. Environ. Microbiol. 75, 3196-3205. doi: 10.1128/AEM.02157-08

Eisenmann, D. M., Dollard, C., and Winston, F. (1989). SPT15, the gene encoding the yeast TATA binding factor TFIID, is required for normal transcription initiation in vivo. Cell 58, 1183-1191. doi: 10.1016/0092-8674(89)90 516-3

Gietz, R. D., and Woods, R. A. (2001). Genetic transformation of yeast. Biotechniques 30, 816-831. doi: 10.2144/01304rv02

Gonzalez Esquivel, D., Ramirez-Ortega, D., Pineda, B., Castro, N., Rios, C., and Perez de la Cruz, V. (2017). Kynurenine pathway metabolites and enzymes involved in redox reactions. Neuropharmacology 112, 331-345. doi: 10.1016/ j.neuropharm.2016.03.013

Goold, H. D., Kroukamp, H., Williams, T. C., Paulsen, I. T., Varela, C., and Pretorius, I. S. (2017). Yeast's balancing act between ethanol and glycerol production in low-alcohol wines. Microb. Biotechnol. 10, 264-278. doi: 10.1111/ 1751-7915.12488

Heyland, J., Fu, J., and Blank, L. M. (2009). Correlation between TCA cycle flux and glucose uptake rate during respiro-fermentative growth of Saccharomyces cerevisiae. Microbiology 155, 3827-3837. doi: 10.1099/mic.0.030213-0 
Hranilovic, A., Gambetta, J. M., Jeffery, D. W., Grbin, P. R., and Jiranek, V. (2020). Lower-alcohol wines produced by Metschnikowia pulcherrima and Saccharomyces cerevisiae co-fermentations: The effect of sequential inoculation timing. Int. J. Food Microbiol. 329, 108651. doi: 10.1016/j.ijfoodmicro.2020. 108651

Hu, R., Lin, L., Liu, T., Ouyang, P., He, B., and Liu, S. (2008). Reducing sugar content in hemicellulose hydrolysate by DNS method: A revisit. J Biobased Mater. Bio. 2, 156-161. doi: 10.1166/jbmb.2008.306

Li, Y., Zhang, Y., Liu, M., Qin, Y., and Liu, Y. (2019). Saccharomyces cerevisiae isolates with extreme hydrogen sulfide production showed different oxidative stress resistances responses during wine fermentation by RNA sequencing analysis. Food Microbiol. 79, 147-155. doi: 10.1016/j.fm.2018.10.021

Liu, N., Wang, D., Wang, Z. Y., He, X. P., and Zhang, B. (2007). Genetic basis of flocculation phenotype conversion in Saccharomyces cerevisiae. FEMS Yeast Res. 7, 1362-1370. doi: 10.1111/j.1567-1364.2007.00294.x

Ljungdahl, P. O., and Daignan-Fornier, B. (2012). Regulation of amino acid, nucleotide, and phosphate metabolism in Saccharomyces cerevisiae. Genetics 190, 885-929. doi: 10.1534/genetics.111.133306

Nevoigt, E., and Stahl, U. (1996). ). Reduced pyruvate decarboxylase and increased glycerol-3-phosphate dehydiogenase [NAD+] levels enhance glycerol production in Saccharomyces cerevisiae. Yeast 12, 1331-1337. doi: 10.1002/ (SICI)1097-0061(199610)12:13<1331::AID-YEA28<3.0.CO;2-0

Nevoigt, E., and Stahl, U. (1997). Osmoregulation and glycerol metabolism in the yeast Saccharomyces cerevisiae. FEMS Microbiol. Rev. 21, 231-241. doi: 10.1016/ S0168-6445(97)00058-2

Otterstedt, K., Larsson, C., Bill, R. M., Stahlberg, A., Boles, E., Hohmann, S., et al. (2004). Switching the mode of metabolism in the yeast Saccharomyces cerevisiae. EMBO Rep. 5, 532-537. doi: 10.1038/sj.embor.7400132

Özcan, S., and Johnston, M. (1999). Function and regulation of yeast hexose transporters. Microbiol. Mol. Biol. Rev. 63, 554-569.

Panozzoa, C., Nawara, M., Suskia, C., and Kucharczyka, R. (2002). Aerobic and anaerobic $\mathrm{NAD}^{+}$metabolism in Saccharomyces cerevisiae. FEBS Lett. 517, 97-102. doi: 10.1016/S0014-5793(02)02585-1

Quiros, M., Rojas, V., Gonzalez, R., and Morales, P. (2014). Selection of nonSaccharomyces yeast strains for reducing alcohol levels in wine by sugar respiration. Int. J. Food Microbiol. 181, 85-91. doi: 10.1016/j.ijfoodmicro.2014. 04.024

Rodríguez, A., De La Cera, T., Herrero, P., and Moreno, F. (2001). The hexokinase 2 protein regulates the expression of the GLK1, HXK1 and HXK2 genes of Saccharomyces cerevisiae. Biochem. J. 355, 625-631. doi: 10.1042/bj3550625

Rolle, L., Englezos, V., Torchio, F., Cravero, F., Ríosegade, S., Rantsiou, K., et al. (2017). Alcohol reduction in red wines by technological and microbiological approaches: A comparative study. Aust. J. Grape Wine R. 24, 62-74. doi: 10. 1111/ajgw.12301

Seong, Y. J., Park, H., Yang, J., Kim, S. J., Choi, W., Kim, K. H., et al. (2017). Expression of a mutated SPT15 gene in Saccharomyces cerevisiae enhances both cell growth and ethanol production in microaerobic batch, fed-batch, and simultaneous saccharification and fermentations. Appl. Microbiol. Biotechnol. 101, 3567-3575. doi: 10.1007/s00253-017-8139-2

Spiropoulos, A., Tanaka, J., Flerianos, I., and Bisson, L. (2000). Characterization of hydrogen sulfide formation in commercial and natural wine isolates of saccharomyces. Am. J. Enol. Viticult. 51, 233-248.
Sporty, J., Lin, S. J., Kato, M., Ognibene, T., Stewart, B., Turteltaub, K., et al. (2009). Quantitation of $\mathrm{NAD}^{+}$biosynthesis from the salvage pathway in Saccharomyces cerevisiae. Yeast 26, 363-369. doi: 10.1002/yea.1671

Tan, S. Z., Manchester, S., and Prather, K. L. (2016). Controlling central carbon metabolism for improved pathway yields in Saccharomyces cerevisiae. ACS Synth. Biol. 5, 116-124. doi: 10.1021/acssynbio.5b00164

Tarazona, S., Garcia-Alcalde, F., Dopazo, J., Ferrer, A., and Conesa, A. (2011). Differential expression in RNA-seq: A matter of depth. Genome Res. 21, 22132223. doi: 10.1101/gr.124321.111

Tilloy, V., Cadière, A., Ehsani, M., and Dequin, S. (2015). Reducing alcohol levels in wines through rational and evolutionary engineering of Saccharomyces cerevisiae. Int. J. Food Microbiol. 213, 49-58. doi: 10.1016/j.ijfoodmicro.2015.06. 027

Tilloy, V., Ortiz-Julien, A., and Dequin, S. (2014). Reduction of ethanol yield and improvement of glycerol formation by adaptive evolution of the wine yeast Saccharomyces cerevisiae under hyperosmotic conditions. Appl. Environ. Microbiol. 80, 2623-2632. doi: 10.1128/AEM.03710-13

Trapnell, C., Roberts, A., Goff, L., Pertea, G., Kim, D., Kelley, D. R., et al. (2012). Differential gene and transcript expression analysis of RNA-seq experiments with TopHat and Cufflinks. Nat. Protoc. 7, 562-578. doi: 10.1038/nprot.2012. 016

Varela, C., Dry, P. R., Kutyna, D. R., Francis, I. L., Henschke, P. A., Curtin, C. D., et al. (2015). Strategies for reducing alcohol concentration in wine. Aust. J. Grape Wine R. 21, 670-679. doi: 10.1111/ajgw.12187

Varela, C., Kutyna, D. R., Solomon, M. R., Black, C. A., Borneman, A., Henschke, P. A., et al. (2012). Evaluation of gene modification strategies for the development of low-alcohol-wine yeasts. Appl. Environ. Microbiol. 78, 60686077. doi: 10.1128/AEM.01279-12

Varela, C., Schmidt, S. A., Borneman, A. R., Pang, C. N. I., Kromerx, J. O., Khan, A., et al. (2018). Systems-based approaches enable identification of gene targets which improve the flavour profile of low-ethanol wine yeast strains. Metab. Eng. 49, 178-191. doi: 10.1016/j.ymben.2018.08.006

Varela, J., and Varela, C. (2018). Microbiological strategies to produce beer and wine with reduced ethanol concentration. Curr. Opin. Biotechnol. 56, 88-96. doi: 10.1016/j.copbio.2018.10.003

Yang, J., Bae, J. Y., Lee, Y. M., Kwon, H., Moon, H. Y., Kang, H. A., et al. (2011). Construction of Saccharomyces cerevisiae strains with enhanced ethanol tolerance by mutagenesis of the TATA-binding protein gene and identification of novel genes associated with ethanol tolerance. Biotechnol. Bioeng. 108, 1776-1787. doi: 10.1002/bit.23141

Conflict of Interest: The authors declare that the research was conducted in the absence of any commercial or financial relationships that could be construed as a potential conflict of interest.

Copyright (c) $2020 \mathrm{Du}$, Liu, Song and Qin. This is an open-access article distributed under the terms of the Creative Commons Attribution License (CC BY). The use, distribution or reproduction in other forums is permitted, provided the original author(s) and the copyright owner(s) are credited and that the original publication in this journal is cited, in accordance with accepted academic practice. No use, distribution or reproduction is permitted which does not comply with these terms. 